(c) 2006 International Press

Adv. Theor. Math. Phys. 10 (2006) 217-237

\title{
Some remarks on fundamental physical $\boldsymbol{F}$-theory
}

\author{
Igor Kriz \\ Department of Mathematics, University of Michigan \\ 2074 East Hall/530 Church Street, Ann Arbor, MI 48109-1043, USA \\ and \\ Michigan Center for Theoretical Physics \\ 3444B Randall Laboratory, University of Michigan \\ 450 Church Street, Ann Arbor, MI 48109-1040, USA \\ ikriz@umich.edu
}

\begin{abstract}
The purpose of this paper is to investigate the possibility of a physical 12-dimensional $F$-theory. We study the question of geometric interaction terms in the $F$-theory Lagrangians. We also introduce a new supergravity multiplet in dimension $(9,3)$ which is based on a particle with 3-dimensional time-like world volume. A construction of signature $(9,3) \quad F$-theory is given using dualities analogous to those considered by Hull, and possible matches of $F$-theory's low energy fields with the $(9,3)$-supergravity field content are given. Finally, preliminary suggestions are made regarding a possible phenomenological compactification of $F$-theory from dimension $(9,3)$ to $(3,1)$.
\end{abstract}

\section{Introduction}

The purpose of this paper is to introduce some new evidence for a fundamental physical 12-dimensional $F$-theory and to exhibit, at least on a

e-print archive: http://lanl.arXiv.org/abs/hep-th/0508046 
preliminary basis, some of its implications. $F$-theory has been first suggested by Vafa [6] and has been subsequently mentioned many times in the literature $[1,7,19,21]$. In some cases, it has been merely suggested as a bookkeeping tool for action terms in lower-dimensional compactifications, in particular in the case of a fiber bundle with elliptic fiber over a type IIB spacetime. Although this suggests that more should be going on, and that a fundamental $F$-theory unifying these contexts should exist, there are difficulties with such a proposal. For example, it is not clear what the theory's low energy limit should be, since supergravities in the classical sense do not exist above dimension 11 .

The purpose of this paper is to investigate these questions and to show new indications that a fundamental $F$-theory, in fact, does exist, while exploring how that would be possible and what it would imply. The first motivation of this project was a previous joint investigation with Sati and coworkers [2-4], where we discovered evidence that $F$-theory is relevant in a different setting, namely $M$-theory and type IIA string theory. There, one does not have an elliptic fibration over 10-dimensional spacetime, but a 12-dimensional manifold with boundary which is a spin cobordism of the spacetime of $M$-theory compactified on $S^{1}$. Witten [11,12] found that this 12 -manifold must be considered when investigating the effective action of $M$-theory. Diaconescu et al. [12] compared the partition function of $M$-theory thus obtained with the partition function of type IIA string theory which is calculated using quantization by $K$-theory. Yet, it did not yet follow from this why $F$-theory action should be considered on this cobordism manifold, and how it relates to the type IIB context. In particular, in the IIB context, certain action terms occur which did not have an obvious analogue in $M$-theory. This concerns, in particular, the lift of the IIB field $G_{5}$.

Jointly with Sati, we more or less accidentally found an extension of the $K$-theoretical partition function $[12,13]$ of type II string theory, which uses elliptic cohomology instead of $K$-theory. The first indication that such function should exist was just the observation [2] that an anomaly $W_{7}\left(X^{10}\right)$ found by [12] is in fact the same as the obstruction to orientability of spacetime $X^{10}$ with respect to elliptic cohomology. When actually defining the function, however, one needs to define a "quadratic structure" (which amounts to a real structure), at which point other obstructions emerge, notably $w_{4}$ or $\lambda$, (depending on whether one uses real-oriented elliptic cohomology or topological modular forms (TMFs)). This suggests that this elliptic partition function has some connection to type I and heterotic string theory, where one sees such anomalies. Trying to interpret this function, we see that it indeed arises as free field approximation of a certain dynamical theory. We eventually concluded that the theory needed is $F$-theory and proposed that an analog of the investigation of [12], which would match the elliptically 
refined partition function of type IIA string theory with the partition function of F-theory, should be possible using Witten's loop version [14] of the Dirac operator. This proposal was recently partially carried out by the Kriz and Xing [5].

In the case of type IIB string theory, the evidence for elliptic partition function is even stronger. It was noted in [12] that a twisted $K$-theory field strength which is the natural extension of the $K$-theory field strength considered there (see also [13]) violates $S$-duality of type IIB. Kriz and Sati $[3,4]$ in fact showed that this cannot be remedied simply by modifying the definition of twisted $K$-theory without introducing other fields. Their investigation suggested that the problem is in the twisting itself and that to remedy it, one needs a theory where the field $H_{3}$ does not introduce a twisting. The natural candidate is again elliptic cohomology (or more precisely the theory of TMFs) where $H_{3}$ "untwists" and merely is represented by multiplication by some element of that generalized cohomology theory. Again, it is a natural suggestion that this partition function should match the partition function of $F$-theory is a theory on 12-dimensional spacetime (although at the present time, we do not have a proof yet). The type of $F$-theory which arises in this case is on $Z^{12}=X^{10} \times E$, where $E$ is an elliptic curve. Modularity of our elliptic partition function is, by the proposal of [4], related to modularity of the first cohomology group $H^{1}(E)$. We further proposed that modularity of the elliptic partition function contains $S$-duality in type IIB string theory.

However, how are the $F$-theories arising in types IIA and IIB related? How would one get a hold of the relevant terms of its action? And what is its low energy limit? These questions are the main subject of the present paper. Actually, a beginning of the discussion of the action is in [5]. There, we first of all explained how it is possible that the $Z^{12}$ which is a spin cobordism of $M$-theory spacetime only seems to have a $G_{4}$-field (a lift of the 4-dimensional field strength in $M$-theory, see [11]), while the action of the $F$-theory which is elliptically fibered on IIB seems to involve a $G_{5}$-field. When investigating the 12-dimensional theory which could have the same partition function as the elliptic refinement of IIA, the natural object to look at was a $G_{4}$-field on the free loop space $L Z^{12}$, which can be investigated using Witten's formula [14] for index on loop space. But a $G_{4}$-field on $L Z^{12}$ produces both a $G_{4}$ and $G_{5}$ on $Z^{12}$. In [5], we were interested mostly in the case when this field is pulled back from $G_{4}$ on $Z^{12}$, in which case we obtain a refinement of the $M$-theory partition function, which, indeed, turns out to match, in an appropriate sense, the elliptic refinement of the IIA partition function. That was the main result of [5]. In the present paper, we investigate the general case, in which the relevant interaction term can be described as $E_{8}$-index on the loop space $L Z^{12}$. In particular, we find that 
there is a quantization condition on $G_{4}$ and $G_{5}$ that they must jointly lift to an $E_{8}$-bundle on $L Z^{12}$, but except that condition, $G_{4}$ and $G_{5}$ are in fact independent fields in $F$-theory, i.e., there is no explicit constraint between them.

Next thing we considered in [5] was how the $F$-theory on a cobordism of $M$-theory spacetime and $F$-theory elliptically fibered on IIB spacetime could be related by duality. We proposed, in fact, such a process: in the case of IIA spacetime $X^{9} \times S^{1}$ subject to $T$-duality, we obtain $M$-theory on $X^{9} \times S^{1} \times S^{1}$. Given appropriate spin structure, we can obtain a spin cobordism $Z^{12}$ which essentially involves only the $S^{1} \times S^{1}$ coordinates. Now the appropriate analogue of $T$-duality shrinks only the boundary (the $M$ theory spacetime) to a space one dimension lower (by contracting one copy of $S^{1}$ to a point). This corresponds to turning $Z^{12}$ to a manifold without boundary. In a suitable case, this is, in fact, the " $T$-dual" $F$-theory fibered over type IIB spacetime. There is, however, by similar arguments, also another type of duality which is a self-duality of $F$-theory without boundary. This duality interchanges the $G_{4^{-}}$and $G_{5}$-fields. This is what makes it feasible to conjecture that there is only one universal $F$-theory rather than separate theories of "types IIA and IIB", and possibly other types.

Now it remains to explain how this would work in physical signatures. We address that in the present paper. Hull [19] considers a IIA*-theory, which is a $T$-dual of IIB-theory on a time-like coordinate of signature $(9,1)$ spacetime. The strong coupling limit is $M^{*}$-theory, which has spacetime of signature $(9,2)$. The cobordism one gets in the last paragraph in that case has signature $(9,3)$. Thus, applying the duality described above, one can construct $F$-theory of signature $(9,3) .{ }^{1}$

The next interesting question is what the low energy limit of such theory might be, given the fact that there is no 12-dimensional supergravity in the conventional sense. We give a proposal for that in the present paper: in a truly physical spacetime of signature $(9,3)$, particles should have 3 -dimensional world volumes with three time-like coordinates. Looking at massless spectra of such type, we get different supersymmetry conditions. In fact, in signature $(9,3)$, we are looking for a supermultiplet which is a representation of a supergroup extension of Spin(6). In this paper, we find in fact a candidate for such supermultiplet which seems to match the fields of $F$-theory and thus could be its low energy limit.

Supposing, finally, that this proposal in fact works, certain interesting possibilities arise. In particular, there is a new type of compactification from

\footnotetext{
${ }^{1}$ The possibility of such theory is in fact mentioned in [19].
} 
dimension $(9,3)$ to dimension $(3,1)$ based on the fact that $(9,3)=3 \cdot(3,1)$. At the end of this paper, we briefly speculate what this could mean for phenomenology. It is possible that instead of splitting spacetime as a direct sum where excess dimensions are "discarded," a $(9,3)$-dimensional space could in fact be represented as a tensor product, where one factor is phenomenological spacetime. Confinement using the 3 -brane in $F$-theory wrapped around a topologically non-trivial submanifold could explain why in the infrared, measurements are approximated by $(3,1)$-dimensional measurements, while however all 12 dimensions are in fact functional as spacetime dimensions. Such theory may have potentially interesting applications, including, for example, a better explanation of distance decay of strong interaction.

The present paper is organized as follows: In Section 2, we shall discuss the action of $F$-theory, in particular the geometric term which we arrived at as a result of the investigation in [5]. In Section 3, we shall discuss the supermultiplet candidate for signature $(9,3)$ supergravity based on a massless particle with three world volume time dimensions. In Section 4, we review the $F$-theory duality discussion from [5] and apply it to the case of physical signatures following the method of Hull [19], constructing an $F$-theory which could produce the field content predicted by the analysis of Section 3. In Section 5, we present the phenomenological applications which could arise from the present theory. Section 6 contains some concluding remarks.

\section{On the action of $F$-theory}

Let us now work to describe the action of $F$-theory in terms of the potentials $A_{3}, A_{4}$, and corresponding field strengths $G_{4}, G_{5}$. There will be, of course, a standard kinetic term of the form

$$
\int_{Z^{12}} G_{4} \wedge * G_{4}+G_{5} \wedge * G_{5}
$$

but there are other interaction terms, including topological and geometric terms, which we need to identify.

We begin by recapitulating known facts. First, let us recall the analysis of the topological Chern-Simons term in $M$-theory, as analysed in $[11,12]$. There, we look at an 11-dimensional manifold $Y^{11}$. Its $M$-theory ChernSimons term should be a topological term and should be expressed by finding a spin 12-manifold $Z^{12}$ whose boundary (with spin) is $Y^{11}$. The leading term is to be

$$
\frac{1}{6} \int_{Z^{12}} G_{4} \wedge G_{4} \wedge G_{4}
$$


but one must adjust the expression so that it does not depend on the choice of the spin cobordism $Z^{12}$. In particular, we want to get 0 on a closed 12-manifold $Z^{12}$. It turns out [11] that the corrected expression is

$$
\frac{1}{6} \int_{Z^{12}} G_{4} \wedge G_{4} \wedge G_{4}+G_{4} \wedge I_{8}
$$

with $I_{8}=\left(p_{2}-\lambda^{2}\right) / 48$. The second term of $(2.3)$ can be interpreted as a 1-loop gravity correction term. When calculating the partition function of $M$-theory, (2.3) actually contributes the phase. This raised the first provocative question to the author and Sati that a 12-dimensional theory may be relevant (although, of course, $F$-theory was originally proposed in a different context by [6]). Now the action (2.3) cannot be the whole story for $F$-theory $Z^{12}$, precisely due to the fact the corresponding phase factor makes sense on a manifold with boundary and vanishes on a closed manifold. In [6], on the other hand, one considers closed manifolds only, which are in fact bundles on type IIB spacetime with fiber an elliptic curve (see also [1,21]).

In [4], jointly with Sati, we showed that in fact very likely considering type IIB string theory as $F$-theory with an elliptic curve fiber is necessary for the consistency of $S$-duality in type IIB string theory in the presence of topological terms. (The breaking of $S$-duality on the level of twisted $K$-theory approximation was actually first noted in [12]; in [3], we showed that this cannot be fixed simply by modifying somehow the definition of twisted $K$-theory in the presence of the same fields.) In the $F$-theory setting, $S$-duality is recovered via a relation between $S$-duality and modularity in $H^{1}(E)$, where $E$ is the fiber. Very interestingly, similar relations in fact also emerged much earlier in Borcherds-Harvey-Moore theory [8-10]. Sati and the author plan to pursue this connection in future work.

In fact, what we did in $[2,3]$ was construct a deformation of the $K$-theory partition function for type IIB and IIA string theory which is modular, using elliptic cohomology instead of $K$-theory, and conjectured (which was later partially confirmed in [5] in the case of IIA) that this is equal to the partition function of $F$-theory (for those phases for which the $F$-theory action indeed reduces to IIA). In fact, to be precise, getting exactly the right function requires the use of the Hopkins-Miller theory of TMFs [5]. We shall return to that point below.

For now, however, let us remark that while exploring that connection, another piece of the picture emerged: to define the elliptic partition function, a 4-dimensional obstruction showed up, which is of a similar nature as obstructions in type I and heterotic string theory. This was our first suggestion that there should be a fundamental $F$-theory which would indeed unify all 10-dimensional string theories. 
Let us now recall what was known about the action of $F$-theory with an elliptic curve fiber. Following essentially the idea of [7] who worked in the case of Calabi-Yau compactifications, we proposed in [4], in the context of $F$-theory compactified on an elliptic curve, the term

$$
\frac{1}{6} \int_{Z^{12}} A_{4} \wedge G_{4} \wedge G_{4}+A_{4} \wedge I_{8}
$$

where $A_{4}$ is a 4 -form potential, $G_{4}$ is 4 -form of $M$-theory lifted to 12 dimensions and $I_{8}=\left(p_{2}-\lambda^{2}\right) / 48$. Specifically, [7] investigated subharmonic expansions of particular form of the potentials $A_{3}, A_{4}$ on $Z^{12}=M^{6} \times \mathrm{CY}$ and showed that then the leading term of (2.4) recovers the expected 6 dimensional coupling term. They also note that for similar reasons, the terms (2.3) must be present.

There are, however, questions about formula (2.4). First of all, [7] note that in their expansion, the potentials $A_{3}$ and $A_{4}$ are not independent. In other words, they conjectured that there must be another independent relation between $A_{4}$ and $G_{4}$. Yet, as far as the author knows, such equation has never been found. In fact, below we will exhibit a curious form of signature $(9,3)$ SUGRA whose fundamental object is a massless particle with 3-dimensional light-like world volume (i.e., a particle moving at the speed of light in three independent time dimensions, which match the three spacetime time dimensions). In that setting, unusual field contents emerge. In particular, in lightcone gauge, we get representations of the group $\operatorname{Spin}(6)$. There, we will see that candidates for both $A_{3}$ and $A_{4}$ occur and are independent (although curiously, $A_{3}$ is in the same representation as the graviton, which leads us to speculate that $A_{3}$ and the graviton merge).

This is evidence that $G_{4}, A_{4}$ indeed should be independent. It is possible that the relation suggested in [7] occurs when looking at a particular sector of the theory. It is not unusual that when looking at compactifications, fields endowed with additional constraints explain terms in the compactified theory: for example, in [12], to get type IIA partition function from $M$ theory on $X \times S^{1}$, one assumes that $G_{4}$ is pulled back from $X$. Nevertheless, we will see below that there is in fact a tie between $G_{4}$ and $G_{5}$, although not exactly in the form of an equation.

We made further progress on answering these questions in [5]. The purpose of that paper was to derive an analogue of [12], which would match the elliptic partition function on $X^{10}$ of type IIA with a partition function of an $F$-theory. Thus, this required defining $F$-theory beyond the case of an elliptic fibration on IIB. Following a proposal in [4], the approach we took 
in [5] was to look at loop versions of the sum of indexes

$$
\frac{I_{E_{8}}}{2}+\frac{I_{R S}}{4}
$$

considered in [12], which is equal to (2.3). Here (2.5) means indices of the Dirac operator twisted by the adjoint $E_{8}$-bundle and the shifted complexified tangent bundle, respectively. The $E_{8}$-bundle is associated with the 4-dimensional integral cohomology class corresponding to $G_{4}$.

By "loop version," we mean the following. The class $G_{4}$ can be pulled back to the loop space $L Z^{12}$. Witten [14] found a way of defining the Dirac operator on loop space. Working in this way requires the condition

$$
\lambda Z=0,
$$

which in fact implies vanishing of all the 4-dimensional anomalies mentioned above. Although this has not been made mathematically rigorous yet, the index is a space with an $S^{1}$-action, and the trace of $q \in \mathbb{C}^{\times}$can be computed using a trace formula, thus giving a power series in $q$, which is a modular form given the condition (2.6). The pullback of the class $G_{4}$ to $L Z^{12}$ can be considered $S^{1}$-equivariant in a neighborhood of fixed loops, so we get a version of $I_{E_{8}}$ which is a modular form. This is what we did in [5]. (To be completely accurate, we essentially neglected the Rarita-Schwinger term and replaced its treatment by just dropping a summand of the loop index which contains $p_{3}$. This is justified in a first approach, as one expects a more complicated boundary behavior in loop spaces, so the exact HořavaWitten analysis of anomaly cancellation will be more difficult to carry out.) In summary, the $E_{8}$-index on loop space $L Z^{12}$ (in the case of field strength pulled back from $Z^{12}$ ) gives the formula

$$
\int_{Z} G\left(\frac{1}{6} G^{2}-5 S_{4} p_{2}\right)
$$

where $S_{\ell}$ is the Eisenstein series

$$
S_{\ell}=S_{\ell}(\tau)=-\frac{B_{\ell}}{2 \ell}+\sum_{n=1}^{\infty}\left(\sum_{d \mid n} d^{\ell-1}\right) q^{n} .
$$

Now we matched in [5] the resulting "phase term" with the phase factor of a variant of the elliptic partition function proposed in [2] on $X^{10}$ of type IIA (where, as above, $Z^{12}$ is a spin cobordism of $X \times S_{R}^{1}$ ). But let us first consider what we computed. To visualize the situation, we can imagine that $G_{4}$ is the field strength associated with a 2-brane $M$, i.e., a 3-dimensional world volume. But now we have considered the propagation of this 2-brane $M$ on $L Z^{12}$, which is equivalent to the propagation of $M \times S^{1}$ on $Z^{12}$. 
We see that therefore we are in fact describing the propagation of a 3-brane, which corresponds to a potential $A_{4}$ or field strength $G_{5}$.

Other observations however must be made. First, the role of $q$ is that the brane, which has one $S^{1}$-factor, propagates along another copy of $S^{1}$, and we take the trace. The $q=e^{2 \pi i \tau}$ measures the moduli parameter of the elliptic curve $E$ which is the product of these two copies of $S^{1}$. Thus, the first observation is that this index is not a topological invariant. It appears, however, reasonable to conjecture that it be a conformal invariant of the brane, since it is a product of a topological invariant and a conformal invariant.

Next, we can in fact only speak of a "phase" if $q$ is in fact real, i.e., the two directions are orthogonal. Otherwise, the factor contributes to amplitude as well as phase. In fact, to even state the comparison with the elliptic partition function in IIA, we must assume that the moduli parameter has special values, where the elliptic curve is defined by an algebraic equation with integral coefficients: in that case, the phase is in fact a topological invariant and (as follows from the results of [5]) does not depend on the choice of $Z^{12}$. This restriction to special values is not completely unexpected, it is a similar effect as e.g., the behavior of special values of L-functions.

Let us return however to the problem of the interaction term of $F$-theory. Given what we learned, we do not really expect the terms $G_{4}$ and $A_{4}$ to be coupled by another equation. That was suggested in [7] for the particular example considered there. However, it is not unusual to impose further restrictions on fields when considering a particular compactification. In [5], we suggested that in fact the potentials $A_{3}$ and $A_{4}$ should be fundamentally independent and only tied by the assumption that they correspond to a joint field strength on loop space:

$$
\left(G_{4}, G_{5}\right)=G \in H^{4}\left(L Z^{12}, \mathbb{Z}\right)
$$

This is consistent with the interpretation of the 4 and 5-dimensional field strength being explained by a 3-brane moving in the loop space. Note however that $L Z^{12}$ is no longer low-dimensional (in fact it is infinite-dimensional), so although an $E_{8}$-bundle has a characteristic class in 4-dimensional integral cohomology, the converse is not true. Therefore, to use index, we need to assume in addition to (2.9) that

$$
G \text { lifts to an } E_{8} \text {-bundle on } L Z^{12} \text {. }
$$

We then have an associated adjoint bundle $\mathcal{V}$ with $G$ on $L Z^{12}$, and the geometric action term in $F$-theory which is the analogue of the elliptic index 
then can be written in general as

$$
\frac{I_{\mathcal{V}}}{2}
$$

We should note that we do not at this point know the correct analogue of the Rarita-Schwinger term. In [5], a natural way to deal with this problem was simply to cut off the $p_{3}$-term, which was sufficient to prove the results stated there.

\section{Signatures and supersymmetry}

As originally noted by Vafa [6], $F$-theory should be considered in physical signatures. One then must ask what is the low energy limit of such theory. Supergravities, in the conventional sense, stop at dimension 11. Nevertheless, when one changes the discussion in certain ways, various higher dimensional scenarios become possible. The purpose of this section is to consider a possibly interesting new case, which is the signature $(9,3)$, when we consider a fundamental particle whose world volume has three time dimensions.

Let us start, following the discussion in [4], by recalling the Clifford algebras in 12 and 11 dimensions with various signatures. A discussion on spinors in different dimensions and with various signatures can be found in [15]. In 12 dimensions, we are interested in $(s, t)$ signatures, with $t=0,1,2,3$. One has symplectic Majorana-Weyl spinors in dimension $(12,0)$, Majorana in dimension $(11,1)$, Majorana-Weyl in dimension $(10,2)$ and symplectic Majorana in dimension $(9,3)$. For the Lorentzian case, $(11,1)$, we have Majorana spinors. In this case, one can try to form a supermultiplet for supergravity formed out of 320 bosons and 320 fermions, but the gravitino and the form sectors of the structure are incompatible [16]. One can then ask whether one can construct supergravity theories with other signatures in 12 dimensions. A general discussion on this can be found in [20], and a proposal in the $(10,2)$ signature can be found in $[17,18]$. There is however a difficulty with supergravity in dimension $(10,2)$ that it contains null states which violate Lorentz covariance.

In the context of [4], both the $(10,2)$ and $(9,3)$ signatures played roles in our conjectures. The main point was compactification of these signatures on an elliptic curve of signature $(1,1)$ and $(0,2)$, respectively, which is conjectured to give type IIA and IIB string theory. The point is that in the $(10,2)$ case, the use of Lorentzian torus breaks modularity ( $S$-duality), which is indeed broken in type IIA. Also the fact that full spacetime Lorentzian invariance is broken in $(10,2)$ does not create a contradiction, since such invariance is also broken by the elliptic curve fibration. In the $(9,3)$ case, 
the $(0,2)$ elliptic curve preserves modularity, which is indeed preserved in type IIB string theory.

Connections with $M$-theory were also proposed in [4], schematically imagining $M$-theory as $F$-theory compactified on a circle. ${ }^{2}$ However, in that sense, the $(10,2)$ signature contains $(10,1)$ SUGRA, so $(10,2) F$-theory indeed appears to contain $(10,1)$ - $M$-theory, while the $(9,3)$ signature scenario seems compatible with higher signature versions of $M$-theory that were found in [19]. In particular, the $(9,2)$ theory in 11 dimensions could be thought of as the dimensional reduction of the $(9,3)$ theory.

Let us examine what kind of supergravity could be the low energy limit of our theory in signature $(9,3)$. In first approximation, the author used a general formula, which says that for $(9,3)$ we can have symplectic-Majorana spinors. It therefore seems possible to propose a particle content for a $N=1$ (9,3)-dimensional supergravity. In lightcone gauge (with three time-like coordinates), the number of helicity states for the gravitino should be (9$3-1) \cdot 2^{(9-3) / 2)}=40$. On the other hand, the graviton has $\left(\begin{array}{l}7 \\ 2\end{array}\right)-1=20$ degrees of freedom, and the potential associated with the $G_{4}$-field strength has $\left(\begin{array}{l}6 \\ 3\end{array}\right)=20$ degrees of freedom, which would seem to give the same number of bosons and fermions.

A more precise analysis, however, reveals a somewhat more subtle picture. First of all, we must discuss in more detail the kind of dynamics we are considering. One can consider particles moving on world lines in (9,3)-space, but this is not what we want. If we did that, in the super-Poincare algebra, the odd part would consist of spinors of the (9,3)-Clifford algebra, which are Majorana, so we would get 64 supercharges. As usual, half of these supercharges must act trivially on a Hilbert representation, but that still leaves 32 supercharges in a Clifford algebra, so the shortest supermultiplet is the spinor which has dimension $2^{16}$. The high number of states seems to indicate that this is probably not the right theory. (Note that in signature $(10,2)$ one gets Majorana-Weil spinors, i.e., 32 independent supercharges, and the shortest supermultiplet has dimension 256, which remains workable.)

However, one can argue that in a truly physical theory, the number of time-like dimension in spacetime and world volume should coincide: in relativity, both notions of time are manifestations of the same entity. From that point of view, in signatures with $k>1$ time-like dimensions, it is natural to work out supergravities describing the dynamics of particles with $k$ time-like

\footnotetext{
${ }^{2}$ The exact discussion is more complicated [4].
} 
world volume dimensions. Assuming that the supergravity particles will be massless, the dimensions which are time-like from world volume point of view will in fact be light-like from the spacetime point of view.

In our case, we shall therefore consider the case when "world-lines" are in fact also 3-dimensional world volumes. This means that every particle possesses three independent relativistic momenta. In a spacetime $V$ with metric of signature $(9,3)$, the momentum then can be encoded by a tensor $V \otimes V \otimes V$. At a definite momentum $p_{1} \otimes p_{2} \otimes p_{3}$, a position operator $q \in V$ acts by multiplication by

$$
\left\langle q, p_{1}\right\rangle\left\langle q, p_{2}\right\rangle\left\langle q, p_{3}\right\rangle
$$

A particle is considered massless when these three momenta are three orthogonal vectors of norm 0 (which is the maximum allowable number of such vectors in signature $(9,3)$ ). Now the three momenta are independent quantum numbers and span a 3-dimensional linear subspace $V$ of the lightcone. By (3.1), displacement along any vector orthogonal to any vector of $V$ acts trivially on the particle. Considering a 3 -element basis $v_{1}, v_{2}, v_{3}$ of $V$, considering each of the vectors $v_{i}$ successively cuts the space of supercharges which are allowed to act non-trivially by $1 / 2$. In the end we end up with $64 / 8=8$ supercharges, which form the total spin representation of Spin(6) (there are two complex conjugate 4-dimensional half-spin representations, which however do not possess a real structure). Thus, the shortest supermultiplet is therefore just has $2^{4}$ elements.

Let us now look at the shortest massless supermultiplet in more detail (see e.g., [24] for a review). We start with irreducible spin representations of Spin $(9,3)$, which gives two complex (not real) representations of dimension 32 each. As mentioned, fixing a non-zero momentum on the lightcone, the dimension of the representation will be cut in $1 / 2$ three times, and we obtain the two 4 -dimensional complex spin representations $4^{+}$and $4^{-}$of $\operatorname{Spin}(6)$. We need a real representation, so the shortest supermultiplet $M$ will be the canonical Clifford module of the Clifford algebra of

$$
4^{+} \oplus 4^{-}
$$

which has dimension

$$
\operatorname{dim}(M)=2^{4}
$$

This is in fact completely analogous to the shortest supermultiplet in dimension $(7,1)$. As a representation of $\operatorname{Spin}(6), M$ decomposes into bosons and 
fermions as

$$
\begin{gathered}
M_{\mathrm{b}}=\mathbf{1}+\mathbf{6}+\mathbf{1}, \\
M_{\mathrm{f}}=4^{+}+4^{-} .
\end{gathered}
$$

Here 6 is the vector multiplet. Now the supermultiplet $M$ can be tensored with a representation $V$ of the transverse part of the symmetry group Spin (6) in search for gravitational supermultiplets. If we restrict ourselves to particle content of spin $\leq 2$, the only possibility is

$$
V=6
$$

or its multiples. Taking (3.2), the supermultiplet

$$
V \otimes M
$$

of dimension 96, which has the following particle content: the fermions are

$$
(V \otimes M)_{\mathrm{f}}=\mathbf{4}^{+}+\mathbf{4}^{-}+\mathbf{2 0}^{+}+\mathbf{2 0}^{-} .
$$

The first two summands are spinors, the second two summands are representations of $\operatorname{Spin}(6)$ of highest weight (110) and (011) (where (010) represents $6,\left(\begin{array}{lll}1 & 0 & 0\end{array}\right)$ and $\left(\begin{array}{lll}0 & 0 & 1\end{array}\right)$ represent $4^{+}$and $\mathbf{4}^{-}$, respectively). Thus, the last two summands constitute the real gravitino representation, as predicted by the general formula.

The bosonic content may be more surprising: we have

$$
(V \otimes M)_{\mathrm{b}}=\mathbf{6}+\mathbf{6}+\mathbf{1}+\mathbf{1 5}+\mathbf{2 0} .
$$

Therefore, we have a scalar (dilaton) and two vectors, the graviton $\mathbf{2 0}$ and an antisymmetric 2-field (or, equivalently 4 -field) $\mathbf{1 5}$. This predicts a potential $A_{2}$ or $A_{4}$, or field strength $G_{5}$ or $G_{3}$ and not $G_{4}$, which seems puzzling. Examining, however, the representations of $\operatorname{Spin}(6)$, we find that

$$
\bigwedge^{3} V \cong \operatorname{Sym}^{2}(V) / \mathbb{R}
$$

i.e., that the graviton and the potential $A_{3}$ transform under the same representation. We therefore conclude that this must be the same particle, which we might call a "gravi-gluon."

We should point out that we have not proved directly that the supermultiplet (3.3) and (3.4) is the correct supergravity multiplet in dimension $(9,3)$, but it is the smallest possible, and the only one which contains only particles with spin $\leq 2$. One can ask how it is possible for this supermultiplet to contain the apparently larger supermultiplet, for example, of type IIB supergravity. The explanation, however, is that we are comparing the states of different objects. In classical supergravity, we have a particle with one light-like world line. In the present $(9,3)$-case, our fundamental object 
is a particle with a 3-dimensional light-like world volume. Thus, this particle has additional degrees of freedom which can absorb any finite number of states. Another way to put it is that the momentum representation of the Poincare group is $V \otimes V \otimes V$ instead of $V$ in the case we are considering.

\section{$4 \quad F$-theory and duality}

In this section, our goal is to examine in more detail the consistency of a $(9,3)$-signature $F$-theory from the point of view of dualities as considered in [19]. In [5], we examined $T$-dualities which link $F$-theory fibered over IIA and IIB without regard to signatures. Let us recall that story first.

In fact, some of this discussion is necessary to explain questions left over in Section 2. For example, we saw that in the $F$-theory related to type IIA, the 12-dimensional spacetime $Z^{12}$ is a manifold with boundary, which is a spin cobordism of the spacetime of $M$-theory. On the other hand, in the context of IIB string theory, we expect $Z^{12}$ to be a closed 12-manifold which is elliptically fibered over $X^{10}$. Yet, these theories should be in a string duality which would lift the $T$-duality between type IIA and IIB. How is that possible?

In [5], we proposed a solution along the following lines: First recall the basic fact that when IIA is considered on a space of the form $X^{9} \times S^{1}$, by shrinking the $S^{1}$ to a point, that coordinate disappears, but a string wrapped around the $S^{1}$ becomes light, which indicates the opening of another dimension, thus giving the $T$-dual IIB-theory on $X^{9} \times S^{1}$. Now it is impossible to apply such $T$-duality naively to higher dimensional theories because of lack of fundamental strings. However, the duality may be recovered by other means. For example, $M$-theory has however 2-branes, and one compactifies $M$-theory on $S^{1} \times S^{1}$ and shrinks this 2-torus to a point, and the 2-brane wrapped on the torus becomes light and new dimension opens up, giving 10-dimensional IIB-theory.

Note that in our settings, where we are using generalized cohomology for flux quantization, there is an additional subtlety, namely that we must take into account spin structure on type IIA and IIB spacetime. Consider type IIA string theory on $X^{9} \times S_{\mathrm{NS}}^{1}$, which is $M$-theory on

$$
X^{9} \times S_{\mathrm{NS}}^{1} \times S_{R}^{1}
$$

Now we know, however, that this is really an approximation of $F$-theory on a spin cobordism $Z^{12}$ between (4.1) and 0. But in the special case (4.1), a 
particular choice of $Z^{12}$ can be proposed, namely

$$
X^{9} \times E^{\prime} \times S_{R}^{1},
$$

where $E^{\prime}$ is a spin cobordism from $S_{\mathrm{NS}}^{1}$ to 0 . Then we can consider a process under which the size of $S_{\mathrm{NS}}^{1}$ shrinks to 0 in the boundary, while preserving the bulk. This corresponds to gluing a disk to $E^{\prime}$. Denoting the corresponding closed surface by $E$ (which can be an arbitrary Riemann surface, in particular an elliptic curve), we get the corresponding bulk $F$-theory on

$$
X^{9} \times E \times S_{R}^{1} .
$$

Thus, we obtain indeed a " $T$-duality" between the $F$-theory with boundary $M$-theory and $F$-theory fibered on IIB spacetime in this case.

In [5], we also noted that there should be another "self- $T$-duality" of the fibered $F$-theory. Consider $F$-theory on

$$
X^{9} \times \prod_{i=1}^{3} S_{R}^{1}
$$

which is a special case of (4.3). Then this theory should have a 2-brane $M_{2}$ and a 3-brane $M_{3}$ where the relationship (2.9) becomes

$$
M_{3}=M_{2} \times S^{1} .
$$

In particular, then $M_{2}$ can be wrapped on

$$
\prod_{i=2}^{3} S_{R}^{1}
$$

and $M_{3}$ on

$$
\prod_{i=1}^{3} S_{R}^{1} .
$$

If we shrink the radius of the first copy of $S_{R}^{1}$ to 0 , then $M_{3}$ will lose a dimension, but $M_{2}$ will expand by the new dimension, and we see then that the system $\left(M_{2}, M_{3}\right)$ is self-dual.

Now let us look at this from the point of view of signatures, as considered in Hull [19]. Hull constructs IIA and IIB-like theories as well as $M$-theory in a variety of signatures. Although these theories pass a number of consistency checks, proposing those theories and then checking their consistency is not the main point of [19]. Rather, the main point is that these theories must exist if we make one simple assumption, namely that in a physical spacetime, the time dimension can be compact (i.e., topologically an $S^{1}$ ). This assumption seems to be widely accepted now; in fact, many arguments 
are only strictly correct if the entire spacetime manifold is compact. Given this assumption, the theories of [19] are simply constructed by applying $T$-duality in the time-like dimensions.

In particular, following [19], if we take a $T$-dual of a signature $(9,1)$ type IIB-theory in the time-like dimension, we obtain a theory in signature $(9,1)$ denoted IIA*. It differs from IIA in that in the low energy action, the signs in the RR-sector are reversed. Accordingly, instead of branes which are world volumes of dimension $(2 k-1,1)$ in type IIA, we have branes which are world volumes of dimension $(2 k, 0)$, i.e., time instantons.

Hull continues to examine the theory IIA*, in particular, its strong coupling limit. He concludes that although the strong coupling limit is 11-dimensional, because of the sign reversal, the additional dimension is in fact time-like, i.e., of signature $(9,2)$. He calls this theory $M^{*}$.

Now let us look at this theory from the point of view of [5]. In particular, our IIA*-theory is on a spacetime of the form

$$
X^{9} \times S_{\mathrm{NS}, t}^{1}
$$

where $X^{9}$ is space-like, and the subscripts NS and $t$ stand for "NeveuSchwarz" and "time-like," respectively. Therefore, $M^{*}$ is on

$$
X^{9} \times S_{\mathrm{NS}, t}^{1} \times S_{R, t}^{1} .
$$

Now $F$-theory is on a spin cobordism of the manifold (4.6) to 0 . This manifold is of the form

$$
X^{9} \times E_{t}^{\prime} \times S_{R, t}^{1}
$$

where $E$ is a 2-dimensional time-like (signature $(0,2)$-) cobordism of $S_{\mathrm{NS}, t}^{1}$ with 0 .

Now let us apply the technique of [5] of shrinking the boundary of $E_{t}^{\prime}$ to a point (while preserving the bulk). In this limit, we obtain a theory on

$$
X^{9} \times E_{t} \times S_{R, t}^{1}
$$

where $E_{t}$ is $E_{t}^{\prime}$ with a disk attached. It is possible to choose $E_{t}$ to be any Riemann surface, in particular

$$
E_{t}=S_{R, t}^{1} \times S_{R, t}^{1},
$$

in which case $(4.8)$ becomes

$$
X^{9} \times \prod_{i=1}^{3} S_{R, t}^{1} .
$$


The spacetime $X^{9} \times S_{R, t}^{1}$ where $S_{R, t}^{1}$ is the first factor (4.10) is now $T$-dual to the original spacetime (4.5) and is therefore of type IIB. Therefore, we have constructed $F$-theory of signature $(9,3)$ fibered over IIB of signature $(9,1)$ by a time-like elliptic curve.

Let us now briefly examine branes in this setting and try to match them to the supergravity sources (3.4). Specifically, let us notice that type IIA* has a $(3,0)$-signature world volume. In the loop space $L Z^{12}$, we obtain world volumes of signatures

and

$$
(3,1),(4,0)
$$

in IIA* $F$-theory. Similarly, the dual world volume in $M^{*}$-theory has $(5,1)$ signature (because $M^{*}$-theory has signature $(9,2)$ ), so in the loop space $L Z^{12}$, we get possible world volumes of signatures

$$
(5,1),(6,1),(5,2) \text {. }
$$

After applying $T$-duality on time-like $S_{\mathrm{NS}}^{1}$ to a point, the world volumes (4.11) will produce 4-dimensional world volumes (picking up an additional time dimension), while the world volumes (4.12) will produce 3- or 5-dimensional world volumes. The world volumes (4.13) will produce 5, 6, 7 or 8-dimensional world volumes. We see that these objects could match all the sources (3.4), plus two non-BPS states in dimensions 7 and 8 .

Let us comment briefly why we are not seeing the particle with 3-dimensional time-like world volume which gives the supergravity in dimension $(9,3)$ we started out with in the first place. Note that one will generally expect to see such fundamental particle as a low energy approximation, but not a direct brane state: for example, type IIA or IIB string theory SUGRA is a low energy approximation of the corresponding string theory, yet the fundamental object of this SUGRA is a particle (with 1-dimensional world line) which is an approximation but not directly a state of the theory. The present situation is analogous. We conjecture, on the basis of field content comparison and possible supergravity supermultiplets, that the low energy limit of signature $(9,3) F$-theory is a supergravity of a massless particle with 3-dimensional world volume as described above, but do not predict such particle to be seen directly as a state of $F$-theory. 


\section{Possible phenomenological predictions of $\boldsymbol{F}$-theory in signature $(9,3)$}

One intriguing aspect of the scenario described above, i.e., a (9,3)-dimensional supergravity based on a massless particle with three time dimensions being the low energy limit of signature $(9,3) F$-theory, is that it offers a possible new phenomenological scenario. The essential point of this observation is the simple fact that $(9,3)=3(3,1)$. It suggests quite a different use of the "excess dimensions" of a higher dimensional description of the universe: we could conjecture that each dimension of $(3,1)$-spacetime is in reality a triplet of dimensions. This does not suggest a "splitting" of dimensions of spacetime in the usual sense, where spacetime would be a direct sum of observable spacetime and excess dimensions, but in fact predicts that spacetime is locally a tensor product

$$
\mathbb{R}^{9,3}=\mathbb{R}^{3,1} \otimes \mathbb{R}^{3}
$$

Therefore, a dimension reduction in this setting means that a dimensional measurement in low energy physics in fact approximates a triplet of measurements. It differs from other approaches to dimensional reductions in the point that in the other types of compactification (such as Kaluza-Klein), there is always a linear combination of dimensions which gives 0 when in terms of observed dimension. Those extra dimensions then have to be explained, and the explanation always seems somewhat unnatural (in particular, one has to ask what physical principle in those models breaks Lorentz invariance and "freezes" the extra dimensions in place). The present model does not have this problem because it does not involve frozen dimensions.

There is another intriguing aspect of the present theory. One difficulty with extending string theory beyond 10 dimensions (including $M$-theory) is the fact that it is not clear what the fundamental object of the theory is (since strings are not critical in dimensions other than 10). In the present theory, we obtained a suggestion that in the low energy limit, the fundamental object is a particle with three time-like world volume dimensions. As noted above, it is very appealing to have a fundamental object whose number of world volume time-like dimensions equals the number of spacetime time-like dimensions. Arguably, this is required of a natural physical theory, extending a basic principle of classical relativity.

We need to explain why, in the infrared, a single measurement of dimension is a good approximation for three measurements. This suggests that there is indeed an approximate relation among the three dimensions in a triplet (from an observation point of view, this is particularly important in the time dimensions). One mechanism which could explain this is that the 
theory in fact has a 3-brane with one time-like dimension (corresponding to the potential $A_{4}$ ). The presence of such stable brane can produce the kind of confinement we need. It might be worth noting that even here, there is a more symmetrical way this may occur than simply splitting off 4 dimensions: suppose, for example, that we have

$$
Z=X_{4} \times X_{4} \times X_{4} .
$$

Then the 3-brane could be wrapped around the diagonal

$$
\begin{aligned}
X_{4} & \subset X_{4} \times X_{4} \times X_{4}, \\
x & \longmapsto(x, x, x),
\end{aligned}
$$

which leaves more symmetries unbroken.

This new scenario is at this point only a proposal. To verify it, one would have to couple the theory to matter and other phenomenological terms. Let us, however, in this paragraph, briefly speculate on at least one phenomenon one may see there. The lesson of string theory seems to be that strong coupling leads to spacetime dimensional expansion. This was first shown by Witten when he discovered that $M$-theory is the strong coupling limit of IIA string theory [22]. For gauge theories and sigma-models, there have been suggestions of such nature [23]. Therefore, one might suspect that the 12dimensional expansion of 4-space should be observed in the strong coupling part of the standard model, which is QCD. In other words, QCD phenomena might lead to local expansion of dimension or observable deviation of $A_{4}$ from the diagonal. Note also that in signature $(p, q)$, the distance behavior of interactions is a decrease with $r^{-p+q}$. For $p=9, q=3$, this is $r^{-6}$, which seems to be enough for confinement, and closer to observation.

\section{Concluding remarks}

The discussion of the present paper leads to the possibility of a phenomenological scenario which is potentially quite different from string-related models proposed before. This is because we are considering a different type of compactification or rather "expansion of one dimension in signature $(3,1)$ into a triplet of dimensions." This scenario is only possible in signature $(9,3)$. To confirm the theory we present here, one needs more precise calculation of $(9,3)$-SUGRA dynamics, although we have reconstructed a substantial part of its action from the effective low energy action of $F$-theory. Another important feature is the "realistic" nature of the dimensional expansion discussed here, which means that the theory has a fundamental object which is a particle with three time-like world volume dimensions, which is equal to the time-like dimensions in spacetime. Phenomenological Lagrangian terms 
would have to be introduced to make more precise predictions, which will be pursued in future work.

\section{Acknowledgments}

I am indebted to H. Sati and H. Xing for collaborations on $F$-theory in the context of the IIA and IIB compactifications. The author is supported by NSF grant DMS 0305853.

\section{References}

[1] R. Friedman, J. Morgan and E. Witten, Vector bundles and F theory, Comm. Math. Phys. 187 (1997), 679-743; arXiv:hep-th/9701162.

[2] I. Kriz and H. Sati, $M$ Theory, type IIA superstrings, and elliptic cohomology, Adv. Theor. Math. Phys. 8 (2004), 345; arXiv:hep-th/0404013.

[3] I. Kriz and H. Sati, Type IIB string theory, S-duality, and generalized cohomology, Nucl. Phys. B 715 (2005), 639; arXiv:hep-th/0410293.

[4] I. Kriz and H. Sati, Type II string theory and modularity, JHEP, 0508 (2005), 038; arXiv:hep-th/0501060.

[5] I. Kriz and H. Xing, Deriving E-theory from F-theory, arXiv:hep-th/0511011.

[6] C. Vafa, Evidence for F-theory, Nucl. Phys. B 469 (1996), 403; arXiv:hep-th/9602022.

[7] S. Ferrara, R. Minasian and A. Sagnotti, Low-energy analysis of $M$ and $F$ theories on Calabi-Yau threefolds, Nucl. Phys. B 474 (1996), 323; arXiv:hep-th/9604097.

[8] R. Borcherds, Automorphic forms on $O_{s+2,2}$ and infinite products, Invent. Math., 120 (1995), 161-213.

[9] J. Harvey and G. Moore, Algebras, BPS states and strings, Nucl. Phys. B 463 (1996), 315; hep-th/9510182.

[10] J. Harvey and G. Moore, On the algebras of BPS states, Comm. Math. Phys. 197 (1998), 489; hep-th/9609017.

[11] E. Witten, On flux quantization in $M$-theory and the effective action, J. Geom. Phys. 22 (1997), 1; arXiv:hep-th/9609122.

[12] E. Diaconescu, G. Moore and E. Witten, E8 gauge theory, and a derivation of K-theory from M-theory, Adv. Theor. Math. Phys. 6 (2003), 1031; arXiv:hep-th/0005090. 
[13] E. Witten, D-Branes and K-theory, JHEP 12 (1998), 019; arXiv:hep-th/9810188.

[14] E. Witten, The index of the Dirac operator in loop space, Proceedings of the Conference on Elliptic Curves and Modular Forms in Algebraic Topology, Princeton, September 1986, Lect. Notes Math., 1326, Springer Verlag, 1986, 161-181.

[15] T. Kugo and P.K. Townsend, Supersymmetry and the division algebras, Nucl. Phys. B 221 (1983), 357.

[16] L. Castellani, P. Fr, F. Giani, K. Pilch and P. van Nieuwenhuizen, Beyond 11-dimensional supergravity and Cartan integrable systems, Phys. Rev. D 26 (1982), 1481.

[17] S. Hewson, On supergravity in $(10,2)$, arXiv:hep-th/9908209.

[18] H. Nishino, Supergravity in $10+2$ dimensions as consistent background for superstring, Phys. Lett. B 428 (1998); arXiv:hep-th/9703214.

[19] C.M. Hull, Duality and the signature of space-time, JHEP 9811 (1998), 017; arXiv:hep-th/9807127.

[20] I. Rudychev, E. Sezgin and P. Sundell, Supersymmetry in dimensions beyond eleven, Nucl. Phys. Proc. Suppl. 68 (1998), 285; arXiv:hep-th/9711127.

[21] A. Sen, F-theory and orientifolds, Nucl. Phys. B 475 (1996), 562-578.

[22] E. Witten, String theory dynamics in various dimensions, Nucl. Phys. B 443 (1995), 85; arXiv:hep-th/9503124.

[23] A.M. Polyakov, Gauge fields and space-time, Int. J. Mod. Phys. A 17S1 (2002), 119; arXiv:hep-th/0110196.

[24] B. De Wit and J. Louis, Supersymmetry and dualities in various dimensions, Proc. NATO Advanced Study Institute on "Strings, Branes and Dualities," Cargese, 26 May-4 June, 1997, eds. L. Baulieu, P. DiFrancesco, M. Douglas, V. Kazakov, M. Picco and P. Windey, NATO ASI C520 Kluwer Ac Publishers, 1999, 33-101; arXiv:hep-th/9801132. 
